\title{
Telescope and site requirements for the next generation of deep galaxy surveys
}

\author{
Eric Steinbring
}

Herzberg Institute of Astrophysics, National Research Council Canada, BC V9E 2E7, Canada email: Eric.Steinbring@nrc-cnrc.gc.ca

\begin{abstract}
A key pursuit of 10-meter-class optical-infrared telescopes is to use deep imaging and spectroscopic surveys to track the evolution of galaxy structure. Future telescopes will continue this quest back to the epoch of the first galaxies, reaching ever fainter structures at ever higher redshifts. Apertures of 20,30,50, and 100 meters equipped with the latest in adaptive optics will look out from the world's foremost observing sites, and incrementally improve on point-source sensitivity. But how will they compare for studying extended structures? Scientific avenues that can be pursued with poorer spatial resolution, but require low backgrounds - for example, tracing the formation history of bulges - might allow for tradeoffs between aperture, site, and cost. To explore this parameter space I use a published model of average seeing at any site, develop a simple telescope performance and cost model, and simulate resultant galaxy images over a wide range of absolute brightness, size, bulge fraction, inclination, and redshift. I present a graphical interface to the model which allows side-by-side visual comparison of a given galaxy for any two observatories. This approach is intuitive and flexible, although probably not well suited for detailed analysis of a particular telescope. I compare observatory cost against the relative accuracy of measured galaxy bulge-to-total ratio, and comment on telescope and site requirements.
\end{abstract}

Keywords. site testing, telescopes, galaxies: fundamental parameters

\section{Introduction}

It is straightforward to predict the point-source sensitivity of a large telescope. And clearly, bigger is better. But what tradeoffs are possible for faint galaxies in the era of Extremely Large Telescopes (ELTs)? There might be little gained from increased resolution when large structures are already marginally resolved with good seeing. Increased aperture means increased cost for glass, steel, and actuators. Putting the same telescope at a better site might make more sense. I have made an initial attempt at a software tool to help explore this parameter space. It employs a graphical interface which allows the user to vary the size, bulge-to-total ratio, and redshift of an artifical galaxy. The galaxy can then be viewed with a toy model telescope of a given aperture, focal ratio, and degree of $\mathrm{AO}$ correction. The cost of the observatory is estimated in order to provide an instant cost-benefit analysis.

\section{World Observatory}

I call my simulation World Observatory because it uses a plausibly general model of observatory image quality. The seeing conditions of mid-latitude sites are estimated using the empirical model of Racine (2005). Only boundary-layer seeing and free-atmosphere seeing are included in this model; scaled by telescope height above the ground and observatory altitude above sea level. Dome seeing is ignored. The free-air seeing at an Antarctic site is taken to be that reported for Dome C in Lawrence et al. (2004) with the 
ground-layer assumed to be the same as a mid-latitutde site of the same elevation. The simulation assumes uncorrected image quality is the seeing at the telescope height scaled to the observed wavelength. Diffraction-limited performance assumes an unobstructed circular aperture. To achieve this, the code assumes one actuator per Fried diameter at the specified wavelength.

The mid-latitude sky background is from a Keck spectrum. For polar sites, the background sky brightness relative to a mid-latitude site is set by a user-given scaling factor. The default is set to a factor of 20 fainter, based on the South Pole results reported by Ashley et al. (1996).

The total observatory cost is estimated from user-defined unit costs for roadway, telescope surface area, enclosure surface area, and AO actuators. There is a premium for polar construction; the default is a factor of 1.5. A new high-altitude or Antarctic site may have better seeing, but it will require a long expensive road. Choosing a larger aperture will increase signal-to-noise ratio (SNR), but combined with the choice of focal ratio it will stretch the budget by forcing a geometrical expansion of the hemispherical dome. Choosing a taller pier improves the seeing, but at the expense of a larger cylindrical base. The cost of the enclosure is taken to be the resulting surface area multiplied by the per-area cost. The cost of adaptive optics is the number of required actuators multiplied by the unit cost. The default inputs give total observatory budgets that seem reasonable, e.g. Keck I and II would cost $\$ 111$ million apiece.

\subsection{Galaxy "Observations"}

The artificial input galaxy can be composed of up to four elements: an $r^{1 / 4}$ bulge, an exponential disk, spiral arms derived from a deconvolved archival Hubble Space Telescope image, and a random distribution of point sources to simulate compact star-formation regions. The intrinsic brightness and bulge-to-total ratio can be controlled by the user, as can the inclination of the disk, and the half-light radius of the bulge. The flux is then scaled with redshift via a template galaxy spectrum and a choice of cosmology. Apparent size also varies with redshift and cosmology.

The galaxy image is generated at high spatial resolution, better than could be achieved with any of the telescopes in the simulation. It is then binned down to either the seeinglimited or diffraction-limited pixel scale of the chosen telescope and convolved with the appropriate point-spread function (PSF). The seeing-limited PSF is a circular twodimensional Gaussian. The AO PSF is composed of a separate Gaussian core and halo. The user-defined Strehl ratio determines what fraction of light falls in the diffractionlimited core. This is very similar to the model PSFs discussed in Steinbring et al. (2005).

Noise is then added to the image. Only sky background is considered; the detector is assumed to be perfect. The simulated galaxies compare well against the Keck AO 1 hour $K$-band images of $z \sim 1$ galaxies in Steinbring et al. (2004), as well as some newer unpublished data taken with the laser.

\subsection{Figures of Merit}

The SNR of the resulting image is calculated using two methods. The first is to subtract the perfect input image from the output and calculate the residual root-mean-square noise. The ratio of this to the observed galaxy peak is called the "structure SNR" because it measures the fidelity in recovering the brightest galaxy structure. The other method is to subtract the image of the disk from the observed galaxy and then measure the flux of the remaining bulge. The error in the result compared to the true value is the "bulge-to-total SNR." 


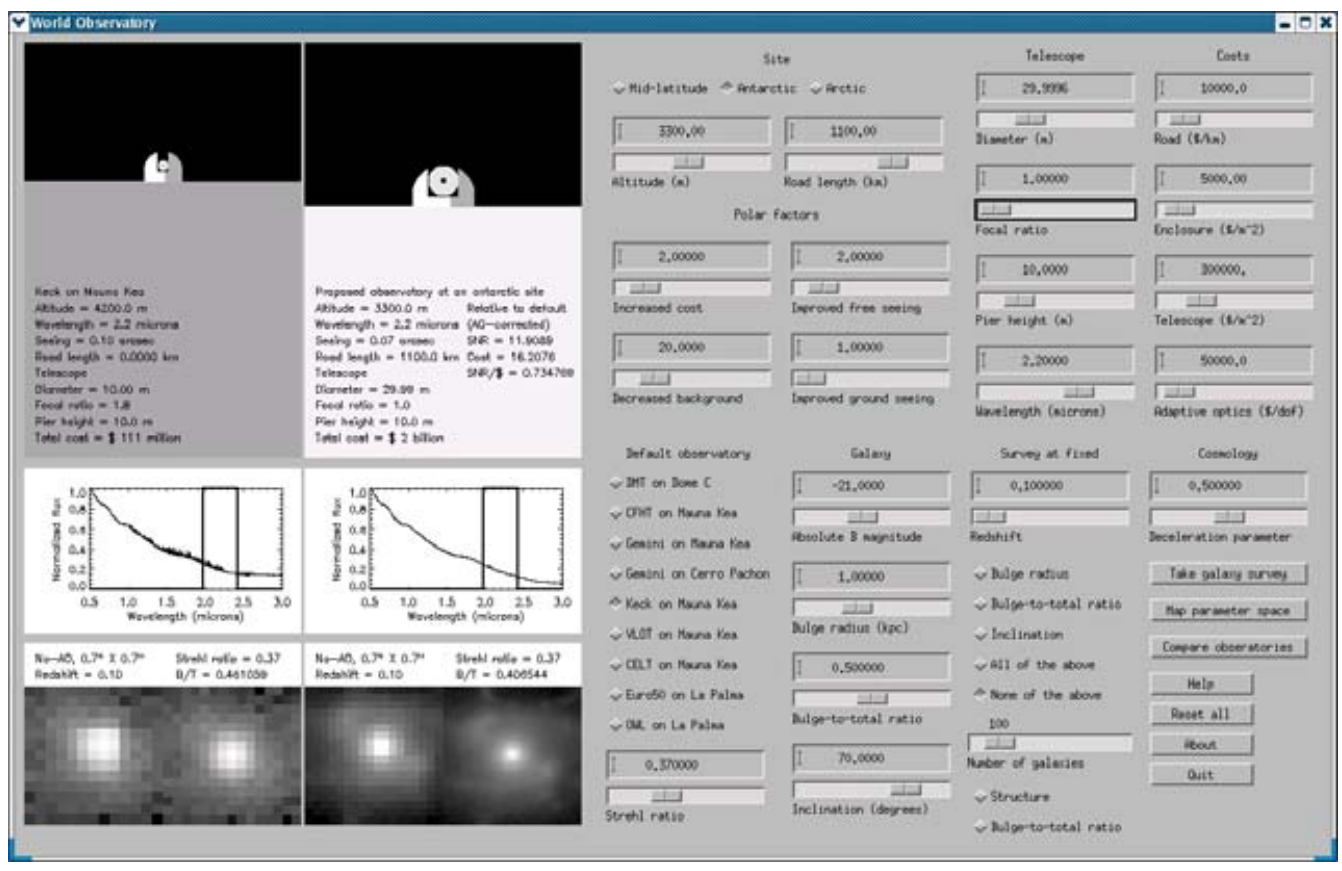

Figure 1. A screen capture of the World Observatory interface. As the user varies the parameters a cartoon of the resulting observatory is presented, and the effect on the image of the galaxy is seen immediately. The image is automatically analyzed and the total cost estimated, which provides a figure of merit in real time for the new observatory.

A figure of merit (FoM) is reported for any choice of parameters. It is the observed SNR (either structure or bulge-to-total) divided by the cost, and the higher this is the better. It is relative to a choice of one among nine existing or plannned observatories. A value greater than unity indicates an advantage over the default observatory. A screen capture of the resulting simulator interface is shown in Figure 1.

\section{Galaxy surveys}

Which observatory is the most cost effective for the next generation of deep galaxy surveys? One can expect that these will routinely reach galaxies with redshifts up to 5 or so. World Observatory can image these distant galaxies with different telescope apertures at either mid-latitude or polar sites, and compare. There is a built-in survey mode for just this purpose.

The averaged results of artificial surveys containing 6500 galaxies with intrisic brightness uniformly distributed between -14 and -26 and redshift between 1 and 5 are shown in Table 1. These random distributions are convenient, but not physically realistic. The other parameters were varied in a similar way, except bulge radius, which was fixed at a challengingly small $0.5 \mathrm{kpc}$. Telescopes with AO on 10, 20, 30, 50, and $100 \mathrm{~m}$ apertures were tested at $K$, each at a 4200-m mid-latitude site and a 3300-m Antarctic site. The results are relative to the mid-latitude $10 \mathrm{~m}$.

The structure SNR improves with aperture, as one expects. The trend is even more dramatic for an Antarctic site. However, this benefit is offset by increased costs, so the structure FoM does not grow with aperture at either site. It is a similar case for bulgeto-total ratio. In fact, an Antarctic 10-m telescope is actually the best performer by a modest amount, reaching a bulge-to-total ratio FoM of 1.1. This last result is not robust 
Table 1. Relative Results for Different Telescope Apertures and Sites

\begin{tabular}{|c|c|c|c|c|c|c|c|c|c|c|}
\hline \multirow{3}{*}{$\begin{array}{l}\text { Diameter } \\
(\mathrm{m})\end{array}$} & \multicolumn{5}{|c|}{ Mid-latitude } & \multicolumn{5}{|c|}{ Antarctic } \\
\hline & \multirow[b]{2}{*}{ Cost } & \multicolumn{2}{|c|}{ Structure } & \multicolumn{2}{|c|}{ Bulge-to-total } & \multirow[b]{2}{*}{ Cost } & \multicolumn{2}{|c|}{ Structure } & \multicolumn{2}{|c|}{ Bulge-to-total } \\
\hline & & SNR & FoM & SNR & FoM & & SNR & FoM & SNR & FoM \\
\hline 10 & 1.0 & 1.02 & 1.02 & 0.98 & 0.98 & 1.6 & 1.33 & 0.81 & 1.80 & 1.10 \\
\hline 20 & 3.6 & 1.71 & 0.47 & 1.35 & 0.37 & 5.6 & 3.57 & 0.64 & 2.17 & 0.39 \\
\hline 30 & 8.1 & 3.10 & 0.38 & 1.55 & 0.19 & 12.4 & 7.55 & 0.61 & 2.39 & 0.19 \\
\hline 50 & 22.2 & 5.23 & 0.24 & 1.80 & 0.08 & 33.4 & 11.45 & 0.34 & 2.70 & 0.08 \\
\hline 100 & 88.4 & 9.32 & 0.11 & 2.29 & 0.03 & 132.2 & 19.35 & 0.15 & 3.21 & 0.02 \\
\hline
\end{tabular}

against variation in costs and background, but it is still worth highlighting. It is an instance where requiring a better site might be more cost effective than building a larger telescope.

\section{Acknowledgements}

I would like to thank René Racine for sharing his analysis of site-testing data prior to publication, and Glen Herriot for helpful discussions about FoMs for ELTs.

\section{References}

Ashley, M.C.B., Burton, M.G., Storey, J.W.V. \& Lloyd, J.P. 1996, PASP 108, 721

Lawrence, J.S., Ashley, M.C.B., Tokovinin, A. \& Travouillon, T. 2004, Nature 431, 278

Racine, R. 2005, PASP 117, 401

Steinbring, E., Faber, S.M., Macintosh, B.A., Gates, E.L. \& Gavel, D. 2005, PASP 117, 847

Steinbring, E., Metevier, A.J., Norton, S.A., Raschke, L.M., Koo, D.C., Faber, S.M., Willmer, C.N.A., Larkin, J.E. \& Glassman, T.M. 2004, ApJS 155, 15

Supplementary archive information is available on-line:

www.journals.cambridge.org./jid_IAU

\section{Discussion}

Crampton: Does your code take operating costs into account?

STEINBRING: No, only capital costs. I'm not sure how to scale operating costs.

MONNET: Is your code "open source"?

STEINBRING: Yes, it is an IDL program which I'll make public. [It is available from the additional materials website of these Procedings or by e-mailing the author.]

DenNefELD: Ultimate sensitivity is strongly dependent on sky brightness. Does your model include the $\mathrm{OH}$ with its variation? I would like to emphasize also the need to include regular $\mathrm{OH}$ monitoring in site testing equipment and weather stations in running observatories: $\mathrm{OH}$ variations are strong (factors of a few) during the night, and are also latitude dependent.

STEINBRING: There is no provision in the code for variation in $\mathrm{OH}$. It allows only relative sky brightness between mid-latitude and polar sites.

MCCARThy: All else being equal, how does cost scale with aperture in your code?

Steinbring: I don't know. Cheaper than the canonical $D^{2.7}$. [The default observatories fall between $D^{1.3}$ and $D^{1.7}$. Stepp, Daggert, \& Gillett (2003, SPIE, 4840, 309) find that the cost of Keck I was $D^{1.2}$ compared to the Mayall $4 \mathrm{~m}$.] 